\title{
Réglementation et dosimétrie individuelle
}

A. BIAU

\subsection{Contexte général}

La réglementation dans le domaine de la protection contre les dangers des rayonnements ionisants en France, comme dans la plupart des pays, évolue dans le temps en fonction de recommandations d'organismes internationaux comme la ICRP et l'Agence internationale pour l'énergie atomique (AIEA).

Le tableau I montre comment les recommandations de la ICRP ont été reprises dans la réglementation française. Il apparaît qu'il faut quelques années de réflexion et de discussions pour transposer les recommandations en textes réglementaires.

TABLEAU I

Chronologie de la transcription des recommandations de la ICRP en droit français.

\begin{tabular}{|c|c|c|c|c|c|}
\hline 1959 & ICRP 2 & 1976 & ICRP 26 & 1990 & ICRP 60 \\
\hline 1960 & & 1977 & & 1991 & \\
\hline 1961 & & 1978 & & 1992 & \\
\hline 1962 & & 1979 & & 1993 & \\
\hline 1963 & & 1980 & Directive $15 / 07 / 80$ & 1994 & \\
\hline 1964 & & 1981 & & 1995 & \\
\hline 1965 & & 1982 & & 1996 & Directive 13/05/96 \\
\hline 1966 & Décret 20/06/66 & 1983 & & 1997 & \\
\hline 1967 & Décret 15/03/67 & 1984 & & 1998 & \\
\hline 1968 & Arrêtés 04/68 & 1985 & & 1999 & \\
\hline 1969 & & 1986 & Décret $02 / 10 / 86$ & 2000 & \\
\hline 1970 & & 1987 & & 2001 & \\
\hline 1971 & & 1988 & Décret $06 / 05 / 88$ & 2002 & Décret 04/04/02 \\
\hline 1972 & & 1989 & & 2003 & Décret 31/03/03 \\
\hline 1973 & & 1990 & & 2004 & Arrêtés 2003-2007 \\
\hline 1974 & & 1991 & & 2005 & \\
\hline 1975 & Décret 28/04/75 & 1992 & & 2006 & \\
\hline 1976 & & 1993 & & 2007 & \\
\hline 1977 & & 1994 & & 2008 & \\
\hline 1978 & & 1995 & & 2009 & \\
\hline 1979 & & 1996 & & 2010 & \\
\hline 1980 & & 1997 & & 2011 & \\
\hline 1981 & & 1998 & Décret 24/12/98 & 2012 & \\
\hline 1982 & & 1999 & Arrêtés 23/03/99 & 2013 & \\
\hline 1983 & & 2000 & & 2014 & \\
\hline
\end{tabular}


Les premières recommandations de la ICRP datent de 1958 , publication $\mathrm{n}^{\circ} 2$ (ICRP, 1959) et ont été prises en compte en France dans les décrets du 20 juin 1966 et du 15 mars 1967 soit après un délai de huit ans.

Les deuxièmes recommandations de la ICRP datent de 1976, publication $\mathrm{n}^{\circ} 26$ (ICRP, 1976) et ont conduit au décret de 1986, soit après 10 ans, mais entretemps la Commission européenne a émis une directive Euratom le 15 juillet 1980, modifiée par une autre directive du 3 septembre 1984. Ces deux directives reprennent les normes de base fixées par la ICRP.

Les troisièmes recommandations de la ICRP en 1991, publication $n^{\circ} 60$ (ICRP, 1991) ont donné lieu à la directive européenne le 13 mai 1996 (EURATOM, 1996) prise en compte dans la réglementation nationale dans le décret du 31 mars 2003, soit 13 ans après.

Les dernières recommandations de la ICRP ont été émises en 2007, publication $n^{\circ} 103$ (ICRP, 2007). À ce jour, la directive européenne qui les prendra en compte ne sera pas publiée avant 2014, ce qui conduit à une transposition dans la réglementation française encore éloignée.

Ces délais ne sont pas dus qu'à des lourdeurs administratives, à chaque fois il y a dans les nouvelles recommandations des évolutions significatives qui remettent en cause certains concepts et qui nécessitent de nombreuses discussions dans les instances administratives.

Les premières recommandations de la ICRP concernaient essentiellement les limites réglementaires. Dans les deuxièmes recommandations, a été introduit le principe ALARA (As Low As Reasonnably Achievable) : ce principe est l'un des trois principes fondamentaux de la radioprotection qui sont la justification des actes à caractère radiologique qui ne doivent être mis en œuvre que s'il n'y a pas d'autre solution, la limitation des doses pour se conformer aux limites réglementaires et l'optimisation qui impose de diminuer les doses aussi bas que raisonnablement possible, compte tenu des facteurs économiques et sociaux. Dans les troisièmes, les limites ont été modifiées pour les travailleurs, le public et, fait nouveau, l'enfant à naître. Dans ses dernières recommandations publiées en 2007, ces limites n'ont pas été modifiées.

La figure 4.1 ci-dessous montre l'évolution des limites réglementaires de dose efficace en considérant que les doses corps entier et doses efficaces sont identiques (stricto sensu la dose efficace n'est apparue qu'en 1990).

\subsection{La réglementation française spécifique à la dosimétrie individuelle}

Les premiers décrets découlant des premières recommandations de la ICRP de 1958 ont donc été le décret du 20 juin 1966 concernant les principes généraux de 


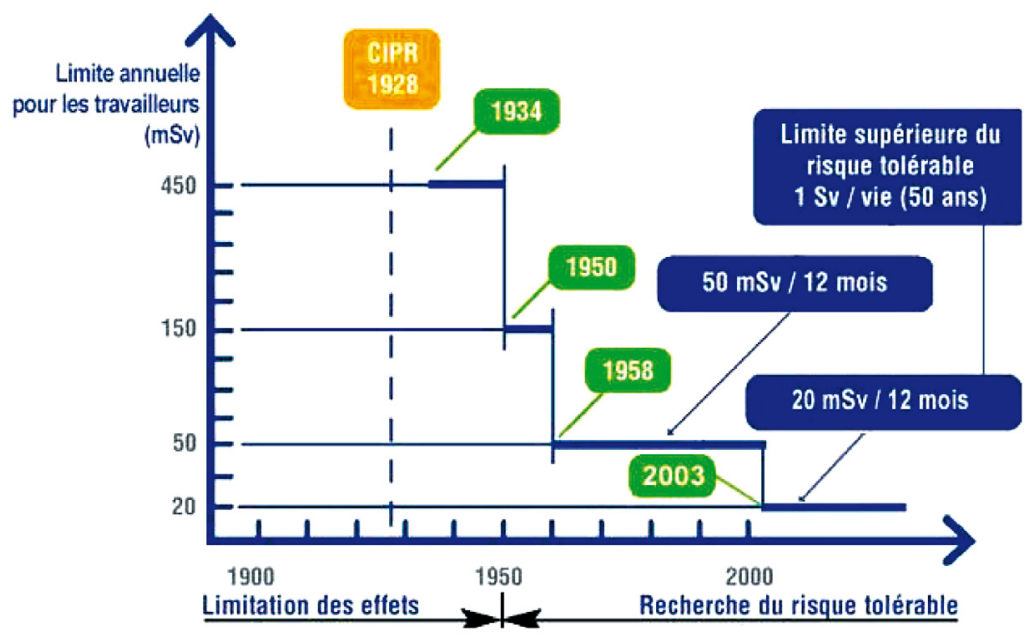

Figure 4.1 - Évolution des limites réglementaires de dose (source IRSN) (OMIRIS, 2004).

la protection contre les dangers des rayonnements ionisants et le décret $\mathrm{n}^{\circ}$ 67-228 du 15 mars 1967 relatif à la protection des travailleurs contre les dangers des rayonnements ionisants. Ce décret, le premier spécifique à la protection des travailleurs sera suivi d'une série d'arrêtés d'application, dont celui du 19 avril 1968 consacré à la dosimétrie individuelle :

\subsubsection{Le décret du 15 mars 1967}

Deux articles sont particulièrement consacrés à la dosimétrie individuelle :

Article 25: «Les travailleurs doivent faire l'objet d'une surveillance individuelle de l'irradiation et de la contamination. Le contrôle des équivalents de dose reçus par les travailleurs directement affectés à des travaux sous rayonnements et exposés au risque d'irradiation externe, doit être assuré au moyen de dosimètres individuels.

Les résultats des contrôles prescrits par le présent article doivent faire l'objet de relevés précis, reportés sur la fiche d'irradiation du dossier médical des intéressés prévu à l'article 30 .

Les résultats des contrôles sont communiqués, sur leur demande, à l'inspecteur du travail et de la main d'œuvre et au médecin inspecteur du travail et de la main d'œuvre. »

Les doses reçues par les travailleurs directement affectés à des travaux sous rayonnements (on disait DATR) doivent faire l'objet d'un contrôle au moyen de 
dosimètres individuels, ce qui exclut les conversions de dosimétrie d'ambiance en dosimétrie individuelle.

L'autre point important est l'aspect «médical » du résultat de dosimétrie individuelle qui sera développé dans l'arrêté du 19 avril 1968.

Article 30 : « Un dossier médical spécial est tenu par le médecin du travail pour chaque travailleur. Mention de ce dossier spécial doit être faite au dossier médical ordinaire de médecine du travail.

Ce dossier doit contenir :

- une fiche de nuisance mentionnant la nature du travail effectué, la nature des rayonnements, la durée des périodes de travail exposant à des rayonnements. La tenue de cette fiche incombe à la personne compétente prévue à l'article 7 de ce décret.

- Une fiche d'irradiation mentionnant les dates et les résultats de contrôle des équivalents de dose reçus.

La tenue de cette fiche incombe au médecin du travail.

Le dossier médical spécial de chaque travailleur doit être conservé pendant la durée de la vie de l'intéressé et, en tout cas, pendant au moins 30 ans après la fin de la période d'exposition aux rayonnements par le service médical de l'entreprise. »

Dans cet article, on introduit la fiche de nuisance et la personne compétente qui deviendra plus tard la personne compétente en radioprotection (PCR) qui existe aujourd'hui.

Il est aussi question du rôle du médecin du travail qui doit tenir à jour la fiche d'irradiation comme élément fondamental du dossier médical spécial. On s'apercevra plus tard, lors de la mise en œuvre d'études épidémiologiques, que les dossiers médicaux spéciaux n'ont pas toujours été tenus à jour et les dossiers eux-mêmes parfois perdus lorsque les travailleurs ont changé plusieurs fois d'entreprise ou lorsque les entreprises dans lesquelles ils ont travaillé ont disparu.

\subsubsection{L'arrêté du 19 avril 1968}

"Conditions d'utilisation des dosimètres individuels destinés au contrôle des équivalents de dose reçus par les travailleurs directement affectés à des travaux sous rayonnements et exposés au risque d'irradiation externe, prescrit par le décret $\mathrm{n}^{\circ}$ 67-228 du 15 mars 1967 relatif à la protection des travailleurs contre les dangers des rayonnements ionisants. » 
Cet arrêté est très important car il a été, à quelques modifications près, en vigueur jusqu'en 1999. Les principaux éléments figurant en annexe de cet arrêté étaient les suivants :

Introduction : «La dosimétrie individuelle ne peut, même dans des conditions expérimentales exceptionnelles, prétendre conduire à une représentation complète et exacte de la répartition spatiale des équivalents de dose reçus par les travailleurs. »

Les limites de la mesure de la dose évoquées au chapitre 2 étaient clairement énoncées.

Domaines d'application : «La dosimétrie individuelle doit être mise en œuvre en cas de risque d'irradiation externe (rayonnements X, gamma, neutrons). Les rayonnements bêta ne sont à prendre en considération, dans le cadre de ce contrôle, que pour des énergies supérieures à $100 \mathrm{keV}$.

La mise en œuvre d'un contrôle dosimétrique individuel dans le cas d'exposition exclusive aux rayonnements alpha et bêta d'énergie maximale faible n'est donc pas recommandée, car les résultats (dans tous les cas négatifs) de ce contrôle pourraient conduire à sous-estimer d'autres risques, en particulier celui de la contamination interne lors de l'utilisation de sources non scellées. »

Ce paragraphe très important a souvent été ignoré ou mal interprété. La dosimétrie des bêtas est très difficile en général sauf pour les électrons de haute énergie émis par le phosphore 32 ou des accélérateurs industriels ou médicaux. Dans ce cas, les doses relevées ne sont pas significatives de l'exposition du corps entier mais de l'épiderme.

Pour les électrons d'énergie inférieure à $100 \mathrm{keV}$, comme ceux du tritium d'énergie moyenne $5,7 \mathrm{keV}$ ou même du carbone 14 d'énergie moyenne $47 \mathrm{keV}$, la dosimétrie individuelle n'est pas justifiée et même trompeuse. En effet, elle peut donner une fausse impression de protection car aucune trace d'exposition ne peut être décelée laissant entendre que l'exposition est nulle. Ces radionucléides sont en général utilisés sous forme de sources non scellées et le vrai risque est l'exposition interne qui peut être correctement évaluée à partir d'analyses radiotoxicologiques des urines.

Choix du dosimètre : «En conclusion, les travailleurs directement affectés à des travaux sous rayonnements (DATR) et exposés au risque d'irradiation externe doivent être munis de dosimètres photographiques individuels dont les conditions de port sont précisées dans les chapitres suivants. Les dosimètres à chambre d'ionisation type « stylo-dosimètre », ou les dosimètres thermo ou photo-luminescents peuvent, bien entendu, être mis en ouvre à titre complémentaire; dans certains cas, l'usage de dosimètres à lecture immédiate est même recommandé. » 
Le choix du dosimètre photographique est imposé, mais les autres techniques ne sont pas ignorées et peuvent permettre l'amélioration des conditions de travail.

Ce monopole du dosimètre photographique a fait l'objet de bien des débats et polémiques. Il faut dire qu'il y avait dans ce choix la volonté de Pierre Pellerin qui, au nom du SCPRI, voyait dans cette technique la possibilité d'assurer une surveillance dosimétrique élargie à tous les milieux notamment hors du domaine nucléaire en proposant un dosimètre simple, peu coûteux (le dosimètre au prix d'un timbre poste). Il est vrai que pour des laboratoires comme ceux de la COGEMA qui avaient les moyens financiers, les compétences et surtout un personnel surveillé limité et localisé, la dosimétrie TLD aurait pu être mise en œuvre mais pour des raisons d'homogénéité la dosimétrie photographique a été imposée à tous les domaines d'activité.

Il faut admettre que le dosimètre photographique a été également utilisé durant de nombreuses années dans plusieurs pays comme le Royaume-Uni, les États-Unis ou le Japon.

Seuil de mesure : "Pour la totalité des rayonnements effectivement utilisés, le seuil de mesure doit être au moins de $0,05 \mathrm{rem}(0,5 \mathrm{mSv})$ et l'étendue minimale de la gamme couverte de 25 rems $(250 \mathrm{mSv})$. »

Ce seuil de 0,5 mSv était en fait un seuil maximal, la formulation n'était pas très heureuse. En fait la plupart des laboratoires utilisaient un seuil de 0,20 mSv. Au SCPRI en 1973, le seuil était de $0,10 \mathrm{mSv}$ (10 millirems) mais était très difficile à « tenir » pour les rayonnements gamma et l'on se trouvait parfois dans la situation où des dosimètres normalement non exposés (dosimètres témoins) se trouvaient avec des doses supérieures à celles des dosimètres portés par des travailleurs !

Il a donc été décidé de revenir à un seuil de $0,20 \mathrm{mSv}$ qui était plus réaliste à la fois pour les basses et les hautes énergies. Les techniques actuelles permettent de présenter des seuils de mesure plus bas mais il faut faire attention, en tout cas pour les dosimètres qui sont dispersés dans des milliers d'établissements, à la mesure de doses qui sont du niveau de celles de l'exposition naturelle. Il est préférable pour les travailleurs de catégorie B potentiellement peu exposés de porter des dosimètres sur une plus longue durée, trois mois par exemple.

Conditions de port du dosimètre : «Le dosimètre est obligatoirement porté pendant les heures de travail. Hors de ces dernières, il est obligatoirement rangé sur un tableau nominatif prévu à cet effet et portant en permanence un dosimètre témoin (ce tableau doit être soigneusement placé à l'abri de toute source de rayonnements ionisants ou de chaleur). La durée de port d'un même dosimètre est le mois civil, sauf accord exprès du Service central de protection contre les rayonnements ionisants. » 
Le rôle du dosimètre témoin est fondamental surtout lorsque le niveau d'exposition des travailleurs est faible; il sert de référence pour les autres dosimètres et tient compte de l'environnement radiologique localement et pendant le transport du laboratoire au lieu de travail à l'aller et au retour.

L'identification du dosimètre: "L'identification du dosimètre au nom du porteur doit être apparente, et l'identification numérique exclure toute équivoque. Le dosimètre est obligatoirement porté à hauteur de la poitrine ou, en cas d'impossibilité à la ceinture. »

Cette obligation d'exclure toute équivoque sur l'identité du porteur s'impose, par contre le fait que l'identification soit apparente a été souvent sujette à caution, lorsque le dosimètre était porté dans une poche du vêtement et encore plus lorsque le travailleur était équipé d'un tablier de protection plombé où dans ce cas il fallait un dosimètre sous le tablier et un autre dessus.

Expression du résultat : "Tout dosimètre ne présentant pas de noircissement décelable doit être considéré comme ayant reçu une dose nulle. »

Cette prescription a été l'objet d'interprétations diverses. Le seuil étant de $0,20 \mathrm{mSv}$, une dose de $0,19 \mathrm{mSv}$ présente un noircissement décelable mais difficilement mesurable en exposition gamma. Au SCPRI, toute dose inférieure au seuil de mesure était considérée comme nulle pour des raisons de simplicité et de cohérence en sachant que dans l'hypothèse peu probable où un individu recevrait $0,19 \mathrm{mSv}$ chaque mois durant 12 mois la dose cumulée serait de $2,28 \mathrm{mSv}$, soit moins que la dose limite pour le public de $5 \mathrm{mSv}$ (jusqu'en 2002). Certains laboratoires ont envisagé d'attribuer la dose égale au seuil dès qu'un noircissement était observé, cette solution qui surestime est discutable également.

Confidentialité des résultats : «Sauf accord exprès du Service central de protection contre les rayonnements ionisants, les résultats mensuels nominatifs doivent être présentés en deux exemplaires sur le formulaire établi par le Service central de protection contre les rayonnements ionisants pour la dosimétrie individuelle. Le premier est adressé au médecin du travail de l'entreprise. Le second, en vue de permettre les intégrations de doses indispensables, est adressé au service de protection contre les rayonnements ionisants. Ces transmissions sont obligatoirement effectuées sous pli cacheté.»

Dans ce paragraphe, est précisé le rôle du SCPRI centralisateur des résultats de dosimétrie individuelle. Il s'agissait en fait d'un archivage des résultats des laboratoires les plus importants mais sans recoupement, la notion de base de données viendra beaucoup plus tard comme nous le verrons au chapitre 13 .

Par ailleurs, la notion de confidentialité des résultats considérés comme éléments du dossier médical spécial selon les termes des paragraphes 25 et 30 du décret du 15 mars 1967 est confirmée. 
Cette notion de résultat d'ordre médical pour la dosimétrie individuelle n'est pas partagée dans d'autre pays et a fait et fait encore l'objet de nombreux débats voire polémiques. Aujourd'hui encore, la dosimétrie active étant accessible et gérée par les PCR, la question se pose pour la dosimétrie passive.

En pratique, les laboratoires ne connaissant, dans la majorité des cas, que l'adresse de l'entreprise abonnée et rarement les coordonnées du médecin du travail, les résultats étaient expédiés à l'adresse de l'entreprise sous pli cacheté à l'attention du médecin du travail avec la mention « confidentiel - résultats médicaux ». Théoriquement, l'enveloppe devait être transmise en l'état au médecin du travail, seul destinataire au sens de la réglementation, mais souvent l'enveloppe était ouverte par l'employeur qui adressait une copie au médecin du travail ou dans certains cas l'employeur ne transmettait rien du tout.

Plus tard, avec l'évolution de l'informatique et la nécessité de créer des bases de données dosimétriques, les adresses des médecins du travail ont été prises en compte et la transmission des résultats plus conforme à la réglementation.

Enfin, dans les années 2000, le système SISERI permettra la constitution plus complète de la base de données dosimétriques réglementaires (voir chapitre 13).

\subsubsection{Le décret du 28 avril 1975}

Ce décret est relatif à la protection des travailleurs contre les dangers de rayonnements ionisants dans les installations nucléaires de base.

Il a été motivé par la progression du nucléaire civil en France et la nécessité de prendre en compte les spécificités de la protection des travailleurs dans ce domaine.

Pour ce qui concerne la surveillance dosimétrique individuelle des travailleurs, les nouvelles prescriptions figurent à l'article 25 .

Article $25-I I$ : «Certains chefs d'établissement peuvent être autorisés par arrêté du ministre chargé du Travail ou du ministre chargé de l'Agriculture à assurer eux-mêmes ladite surveillance (dosimétrie externe). Dans ce cas, les résultats sont communiqués au service central de protection contre les rayonnements ionisants qui vérifie la qualité des mesures effectuées et les confirme par des mesures périodiques. »

Article 25 - III : «Dans le cas où l'employeur des travailleurs n'est pas le chef d'établissement ou qu'il ne bénéficie pas de l'autorisation prévue au II ci-dessus, cet employeur est tenu de faire effectuer la surveillance de l'exposition externe et interne par le service central de protection contre les rayonnements ionisants, ou par un organisme agréé à cet effet par les ministères chargés du Travail et de l'Agriculture après avis du service central de protection contre les rayonnements ionisants. » 
Jusqu'à, la parution de ce décret, l'employeur pouvait faire assurer la surveillance dosimétrique individuelle par l'organisme de son choix sous réserve qu'il se conforme aux termes de l'arrêté du 19 avril 1968.

À partir de ce décret, les exploitants sont officiellement autorisés à assurer cette surveillance pour leurs propres agents, sous réserve de vérifications par le SCPRI. À partir de cette date des intercomparaisons seront organisées chaque année sur les sites de CEA, COGEMA et EDF (voir chapitre 12).

Pour ce qui concerne les agents d'entreprises extérieures dont le nombre devenait de plus en plus important, la surveillance devra être effectuée par le SCPRI.

\subsubsection{Le décret $n^{\circ} 86-1103$ du 2 octobre 1986}

Il concerne la protection des travailleurs contre les dangers des rayonnements ionisants.

Ce décret abroge le décret du 15 mars 1967 et transpose les recommandations de la ICRP de 1976 via la Directive européenne 84-467.

Les limites de dose restent les mêmes, s'appliquent sur 12 mois glissants et sont exprimées en équivalent de dose :

- corps entier en profondeur : $50 \mathrm{mSv}$;

- peau, mains : $500 \mathrm{mSv}$;

- cristallin : $150 \mathrm{mSv}$;

- abdomen femme enceinte : $10 \mathrm{mSv}$ (entre le jour de la déclaration au médecin du travail et l'accouchement).

Les nouveautés concernent la classification des travailleurs en catégories A et B et l'attribution par le médecin du travail d'une carte de suivi médical pour les travailleurs de catégorie A.

Les modalités de la mise en œuvre de la surveillance dosimétrique individuelle sont inchangées et restent celles définies dans l'arrêté du 19 avril 1968.

\subsubsection{Les décrets $n^{\circ}$ 98-1185 et $n^{\circ} 98-1186$ du 24 décembre 1998 modifiant les décrets $n^{\circ} 75-306$ du 28 avril 1975}

Ces décrets sont relatifs à la protection des travailleurs contre les dangers des rayonnements ionisants dans les installations nucléaires de base et $\mathrm{n}^{\circ}$ 86-1103 du 2 octobre 1986 relatif à la protection des travailleurs contre les dangers des rayonnements ionisants.

Ces décrets ont été pris en application de la Directive 90/64 EURATOM concernant la protection opérationnelle des travailleurs exposés au risque de rayonnements au cours de leur intervention en zone contrôlée. 
Ils seront suivis de l'arrêté du 23 mars 1999 sur les conditions de mise en œuvre de la dosimétrie passive et de la dosimétrie opérationnelle.

\subsubsection{L'arrêté du 23 mars 1999}

Cet arrêté précise les règles de la dosimétrie externe des travailleurs affectés à des travaux sous rayonnements en application des articles 20 bis et 225-I du décret du 28 avril 1975 modifié et des articles 31 bis et 34.1 du décret du 2 octobre 1986 modifié.

Toutes les modalités de mise en œuvre de la dosimétrie passive actuellement en vigueur sont décrites dans cet arrêté. Les éléments principaux sont les suivants :

Article 3 : «L'arrêté du 19 avril 1968 précisant les conditions d'utilisation des dosimètres individuels destinés au contrôle des équivalents de dose reçus par les travailleurs affectés à des travaux sous rayonnements et exposés au risque d'irradiation externe est abrogé. »

Annexe I - Champ d'application : «La dosimétrie individuelle doit être mise en œuvre en cas de risque d'exposition externe (rayons X, gamma, neutrons). Le rayonnement bêta n'est à prendre en considération, dans le cadre de ce contrôle, que pour des énergies moyennes supérieures à $100 \mathrm{keV}$.

La présence d'un dosimètre d'ambiance à un poste de travail ne dispense en aucun cas du port d'un dosimètre individuel pour l'agent affecté à ce poste. »

Le texte reprend des dispositions de l'arrêté du 19 avril 1968 en les précisant en ce qui concerne l'énergie des bêtas (100 keV d'énergie moyenne) et la nécessité du port d'un dosimètre individuel qui ne peut pas être remplacé par un dosimètre d'ambiance.

Annexe II - Choix des méthodes de dosimétrie : «Une bonne utilisation d'une technique dosimétrique implique d'une part, que cette technique soit validée et, d'autre part, que les procédures d'exploitation et/ou les conditions d'utilisation soient respectées sous la responsabilité de l'employeur. »

C'est la fin du monopole de la dosimétrie photographique qui s'explique par la qualité des techniques alternatives et également par la fin annoncée de la fabrication d'émulsions photographiques par les grands industriels plutôt orientés sur les technologies de photographie numérique.

\section{Annexe III - Spécifications techniques des dosimètres}

- Critères techniques communs applicables à tout type de dosimètre : «Le dosimètre est individuel et nominatif. L'identification du porteur doit exclure toute équivoque. 
Par convention, l'équivalent de dose ainsi mesuré est assimilé à la dose reçue par le corps entier (dose efficace).

Le dosimètre doit être porté sous les équipements de protection individuelle lorsque leur utilisation est nécessaire dans une opération donnée.

Compte tenu des performances actuelles en matière de mesure des rayonnements bêtas ou neutrons, les résultats correspondants seront assimilés à des données complémentaires relatives à l'exposition.

Tout équivalent de dose enregistré par le dosimètre est réputé reçu par le porteur jusqu'à preuve formelle du contraire.

\section{Le médecin du travail se réfère à la dosimétrie passive}

Ces dispositions existaient déjà dans l'arrêté du 19 avril 1968 mais elles sont ici plus explicites notamment sur le port du dosimètre sous les équipements de protection et la notion de données complémentaires pour les doses bêta et neutron. Cette notion de données complémentaires est un peu choquante pour le puriste en dosimétrie car les doses bêta et neutrons concourent comme les autres composantes à la dose efficace mais encore faut-il que ces composantes puissent être mesurées avec la même qualité que pour les X et gamma. Lorsque c'est possible, dans des laboratoires du CEA ou d'AREVA par exemple, où la surveillance dosimétrique est adaptée aux conditions particulières de travail, les doses bêta et neutrons peuvent être directement prises en compte dans la dose efficace avec l'accord du médecin du travail.

La notion de confidentialité des résultats de dosimétrie passive est confirmée, comme nous le verrons au chapitre consacré aux bases de données et SISERI.

- Caractéristiques de la dosimétrie en temps différé ou "dosimétrie passive »: « Pour l'ensemble des rayonnements susceptibles d'être mesurés, le seuil de mesure doit être au plus de $0,2 \mathrm{mSv}$ et l'étendue de la gamme couverte de $500 \mathrm{mSv} . »$

Le seuil maximal de mesure passe de 0,5 à $0,2 \mathrm{mSv}$ logiquement, car ce seuil représente le centième de la dose maximale admissible de $50 \mathrm{mSv}$ depuis le décret du 15 mars 1967 et qui va devenir $20 \mathrm{mSv}$ en mars 2003 en application de la Directive EURATOM 96/29.

Le tableau II présente l'évolution des seuils de mesure utilisés par les différents laboratoires de 1968 à 2010. Il apparait clairement que les seuils de mesure affichés dans la présentation des résultats ont toujours été inférieurs ou égaux au seuil réglementaire de $0,50 \mathrm{mSv}$ de 1968 à 1998 et de $0,20 \mathrm{mSv}$ depuis 1999. À partir de 2008 après la disparition du dosimètre photographique, les techniques utilisées permettent d'afficher des seuils de mesure très inférieurs au seuil réglementaire de $0,20 \mathrm{mSv}$. 
A. BIAU

Tableau II

Seuils de mesure $(\mathrm{mSv})$ selon les laboratoires (dosimétrie $\mathrm{X}$ et $\mathrm{\gamma})$.

\begin{tabular}{|c|c|c|c|c|c|c|c|c|c|c|}
\hline Année & 0,05 & 0,10 & 0,15 & 0,20 & 0,25 & 0,30 & 0,35 & 0,40 & 0,45 & 0,50 \\
\hline 1968 & & (2) & (5) & (3) (4) & & & (1) & & & $\bullet$ \\
\hline 1969 & & (2) & (5) & (3) (4) & & & (1) & & & $\bullet$ \\
\hline 1970 & & (2) & (5) & (3) (4) & & & (1) & & & $\bullet$ \\
\hline 1971 & & (2) & (5) & (3) (4) & & & (1) & & & $\bullet$ \\
\hline 1972 & & (2) & (5) & (3) (4) & & & (1) & & & $\bullet$ \\
\hline 1973 & & (2) & (5) & (3) (4) & & & (1) & & & $\bullet$ \\
\hline 1974 & & (2) & (5) & (3) (4) & & & (1) & & & $\bullet$ \\
\hline 1975 & & & (5) & (2) (3) (4) & & & (1) & & & $\bullet$ \\
\hline 1976 & & & (5) & (2) (3) (4) & & & (1) & & & $\bullet$ \\
\hline 1977 & & & (5) & (2) (3) (4) & & & (1) & & & $\bullet$ \\
\hline 1978 & & & (5) & (2) (3) (4) & & & (1) & & & $\bullet$ \\
\hline 1979 & & & (5) & (2) (3) (4) & & & (1) & & & $\bullet$ \\
\hline 1980 & & & (5) & (2)(3) (4) & & & (1) & & & $\bullet$ \\
\hline 1981 & & & (5) & (2) (3) (4) & & & (1) & & & $\bullet$ \\
\hline 1982 & & & (5) & (2) (3) (4) & & & (1) & & & $\bullet$ \\
\hline 1983 & & & (5) & (2) (3) (4) & & & (1) & & & $\bullet$ \\
\hline 1984 & & & (5) & (2) (3) (4) & & & (1) & & & $\bullet$ \\
\hline 1985 & & & (5) & (2) (3) (4) & & & (1) & & & $\bullet$ \\
\hline 1986 & (3) $(X)$ & & (5) & (2) (3) (4) & & & (1) & & & $\bullet$ \\
\hline 1987 & (3) $(X)$ & & (5) & (2) (3) (4) & & & (1) & & & $\bullet$ \\
\hline 1988 & (3) $(X)$ & & (5) & (2)(3)(4) & & & (1) & & & $\bullet$ \\
\hline 1989 & (3) $(X)$ & & (5) & (2) (3) (4) & & & (1) & & & $\bullet$ \\
\hline 1990 & (3) $(X)$ & & (5) & (2) (3) (4) & & & (1) & & & $\bullet$ \\
\hline 1991 & (3) $(X)$ & & (5) & (2) (3) (4) & & & (1) & & & $\bullet$ \\
\hline 1992 & (3) $(X)$ & & (5) & (2)(3) (4) & & & (1) & & & $\bullet$ \\
\hline 1993 & (3) $(X)$ & (4) & (5) & (2)(3) & & & (1) & & & $\bullet$ \\
\hline 1994 & (3) $(X)$ & (4) & (5) & (2) (3) & & & (1) & & & $\bullet$ \\
\hline 1995 & (3) $(X)$ & (4) & (5) & (2)(3) & & & (1) & & & $\bullet$ \\
\hline 1996 & (3) $(X)$ & (4) & (5) & (2) (3) & & & (1) & & & $\bullet$ \\
\hline 1997 & (3) $(X)$ & (4) & (5) & (2) (3) & & & (1) & & & $\bullet$ \\
\hline 1998 & (3) $(\mathrm{X})$ & (4) & (5) & (2) (3) & & & (1) & & & $\bullet$ \\
\hline 1999 & (3) & (4) & (5) & (2) 1 & $\bullet$ & & & & & \\
\hline 2000 & (3) & (4) & (5) & (2) (1) & $\bullet$ & & & & & \\
\hline 2001 & (3) & & (5) & (2) (1) (4) & $\bullet$ & & & & & \\
\hline 2002 & (3) & & (5) & (2) (1) (4) & $\bullet$ & & & & & \\
\hline 2003 & (3) & & (5) & (2) (1) (4) & $\bullet$ & & & & & \\
\hline 2004 & (3) & & (5) & (2) (1) (4) & $\bullet$ & & & & & \\
\hline 2005 & (3) (4) & & (5) & (2) (1) & $\bullet$ & & & & & \\
\hline 2006 & (3) (4) & & (5) & (2) 1 & $\bullet$ & & & & & \\
\hline 2007 & (3) (4) & (5) & & (2) (1) & - & & & & & \\
\hline 2008 & (3) (4)(2) & (5) & & & $\bullet$ & & & & & \\
\hline 2009 & (3) (4)(2) & (5) & & & $\bullet$ & & & & & \\
\hline 2010 & (3) (4)(2) & (5) & & & $\bullet$ & & & & & \\
\hline
\end{tabular}

- Seuil réglementaire (1) Seuil CEA/IPSN/IRSN (2) Seuil SCPRI/OPRI/IRSN (3) Seuil LCIE/Landauer (3) $(\mathrm{X})$ seuil LCIE pour les rayons $\mathrm{X}$ (4) COGEMA/AREVA (5) Défense Nationale 
La gamme couverte passe de 250 à $500 \mathrm{mSv}$ pour tenir compte des progrès techniques mais cette disposition a un intérêt limité car les doses supérieures à $100 \mathrm{mSv}$ sont heureusement rarissimes et lorsque l'on atteint de tels niveaux, on se trouve en situation accidentelle et dans ce cas, la dosimétrie biologique est bien plus représentative que la dosimétrie physique pour apprécier la dose réellement reçue par la victime. La dosimétrie biologique a fait de grands progrès ces dernières années et permet d'évaluer des doses comprises entre 50 et $100 \mathrm{mSv}$ mais nécessite des mesures longues et minutieuses qui prennent quelques jours. Elle doit donc être réservée à des incidents ou à des suspicions d'incidents concernant des personnes dépourvues de dosimètre.

Dans le reste de cette annexe figurent des dispositions qui existaient déjà dans l'arrêté du 19 avril 1968 concernant la résistance aux nuisances chimiques, la qualité de la réponse en énergie, la rigueur des étalonnages, la nécessité du dosimètre Témoin.

Les nouveautés concernent essentiellement la gestion des données de la dosimétrie passive et opérationnelle sur lesquelles nous reviendrons au chapitre 8 .

Au paragraphe 4.2, La gestion des données de dosimétrie passive, «Le traitement de la dosimétrie passive est effectuée par l'OPRI, par un laboratoire agrée ou par les établissements bénéficiant d'une autorisation » est confirmée la notion d'agrément pour les laboratoires de dosimétrie autres que l'OPRI. Cette disposition s'appliquera ensuite à l'IRSN lorsqu'il remplacera l'OPRI.

\subsubsection{Le décret $n^{\circ} 2003-296$ du 31 mars 2003}

Ce décret est relatif à la protection des travailleurs contre les dangers des rayonnements ionisants. Il est la transposition en droit français de la Directive 96/29 EURATOM reprenant les recommandations de la ICRP de 1990.

Les discussions ont été longues en raison de quelques points délicats, notamment la limite de doses pour les travailleurs qui, dans la Directive, était de $100 \mathrm{mSv}$ en 5 ans, et qui a été fixée à $20 \mathrm{mSv}$ sur 12 mois dans le décret.

L'autre point sensible a été la limite pour les femmes enceintes qui était de $10 \mathrm{mSv}$ à l'abdomen et, dans la directive, de $1 \mathrm{mSv}$ pour l'enfant à naître. Cette notion d'enfant à naître n'existait pas dans la réglementation française et posait des questions philosophiques ou éthiques. Finalement le texte français s'est conformé à la formulation de la directive.

Sur le plan proprement dosimétrique c'est un concept difficile à assumer et certains pays se sont contentés de définir une limite dérivée au niveau de l'abdomen de la femme enceinte ( $2 \mathrm{mSv}$ par exemple). 
Enfin, voici les points marquants concernant la surveillance dosimétrique individuelle.

\subsubsection{L'article $R$ 231-76}

Limites de doses sur 12 mois glissants :

- dose efficace : $20 \mathrm{mSv}$;

- dose mains, peau, avant-bras : $500 \mathrm{mSv}$;

- cristallin : $150 \mathrm{mSv}$.

\subsubsection{L'article $R$ 231-77}

«En cas de grossesse, les dispositions sont prises pour que l'exposition, dans son emploi, de la femme enceinte soit telle que l'exposition de l'enfant à naître, pendant le temps qui s'écoule entre la déclaration de grossesse et le moment de l'accouchement soit aussi faible que raisonnablement possible, et en tout cas audessous de $1 \mathrm{mSv}$.

\subsubsection{L'article R 231-80}

«S'agissant de l'exposition externe, la mesure de référence utilisée pour vérifier le respect des valeurs limites repose sur la dosimétrie passive...»

Par ailleurs ce décret qui est directement intégré au Code du Travail comprend de nombreuses dispositions concernant la gestion des données qui figuraient dans l'arrêté du 23 mars 1999 et qui sont confirmées et développées (chapitre 13).

\section{RÉFÉRENCES}

EURATOM (1996) Directive EURATOM n 96/29 du 13 mai 1996.

ICRP Publication 2 (1959) Recommendations of the International Commission on Radiological Protection, Pergamon Press.

ICRP Publication 26 (1976) Recommendations of the International Commission on Radiological Protection, Oxford Pergamon Press.

ICRP Publication 60 (1991) Recommendations of the International Commission on Radiological Protection, Pergamon Press.

ICRP Publication 103 (2007) Recommendations of the International Commission on Radiological Protection, Oxford Elsevier.

OMIRIS (2004) CD Rom réalisé sous l'égide de la Fédération des enseignants en Radiologie, Radiopathologie et Radioprotection (FE3R). 